SLAC-PUB-11572

November 2005

\title{
Recent and Future Observations in the X-ray and Gamma-ray Bands: Chandra, Suzaku, GLAST, and NuSTAR
}

\author{
Greg Madejski \\ Stanford Linear Accelerator Center and KIPAC, Stanford CA 94305, USA
}

\begin{abstract}
.
This paper presents a brief overview of the accomplishments of the Chandra satellite that are shedding light on the origin of high energy particles in astrophysical sources, with the emphasis on clusters of galaxies. It also discusses the prospects for the new data to be collected with instruments recently launched - such as Suzaku - or those to be deployed in the near future, and this includes GLAST and NuSTAR.
\end{abstract}

Keywords: High energy astrophysics; X-ray and gamma-ray instruments

PACS: $95.55 ; 98.54 ; 98.65$

\section{INTRODUCTION}

The last several years can be truly called the "golden era" of high energy astrophysics. At the time of this meeting, Chandra and XMM-Newton were conducting imaging observations in the soft X-ray band; RXTE was measuring the timing properties of variable celestial X-ray sources; Integral covered the hard X-ray and soft gamma-ray regime; and Swift, just launched, was effectively discovering and measuring properties of gamma-ray bursts. All this resulted in tremendous advancements of our understanding of sources of high energy radiation - shedding light on the physical processes responsible for the particle acceleration in the Universe. This paper focuses on selected results derived from Chandra data, but also presents the prospects for the near future - and this includes the recently launched Suzaku, and the approved satellite missions GLAST and NuSTAR.

\section{CHANDRA X-RAY OBSERVATORY}

Now entering the seventh year of its operation, Chandra X-ray Observatory was launched by the Space Shuttle into a deep, highly eccentric orbit which takes about 2 2/3 days to complete. Chandra telescope is perhaps the most sophisticated X-ray optical system ever built, capable of imaging at the point-spread function better than $0.5^{\prime \prime}$. The focal plane instruments include the Advanced CCD Imaging Spectrometer (ACIS), an imaging instrument affording moderate-resolution $(\sim 100 \mathrm{eV})$ spectral capability, with the combined mirror+detector effective area peaking at $\sim 700 \mathrm{~cm}^{2}$. The High Resolution Camera, a micro-channel plate detector, is capable of highest spatial resolution while still providing some spectral information. In addition, Chandra can 

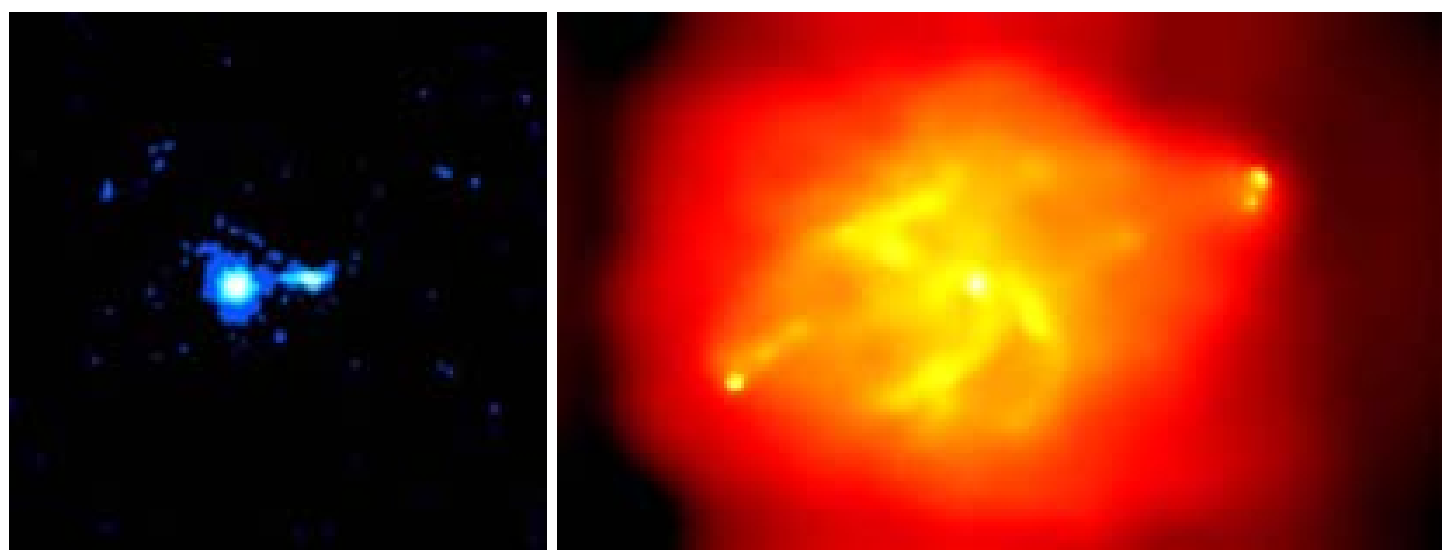

FIGURE 1. Left: X-ray image of the quasar PKS 0637-752, revealing the kpc-scale X-ray emitting jet (data published in Schwartz et al. 2000; figure credit: Chandra X-ray Center and NASA). Right: Chandra X-ray image of the radio galaxy Cyg A, revealing X-ray jets (Figure source: Chandra X-ray Center; figure credit: NASA; data published by Wilson, Young, and Shopbell 2000)

perform high-resolution X-ray spectroscopy, via the use of low- as well as mediumand high-energy transmission gratings, read out by the Chandra imaging detectors. The overall bandpass of the telescope spans $\sim 0.2-10 \mathrm{keV}$.

Over six years of operation, Chandra conducted a wide variety of scientific investigations. Within our Galaxy, those range from studies of planets, star forming regions, normal stars, supernova remnants, binary accreting systems, globular clusters, as well as the Galactic Center. Outside of our Galaxy, it also studied many normal and active galaxies, clusters of galaxies, and the content of the diffuse intergalactic medium, but the most important and unique study was that of the Cosmic X-ray Background (CXB). Observations conducted with the Chandra and XMM-Newton satellites clearly indicated that the main contribution to the $\mathrm{CXB}$ in the $2-10 \mathrm{keV}$ band is indeed due to the superposition of unresolved active galactic nuclei (AGN), but at least the XMM data indicate that the situation is less clear regarding the highest energies accessible to Chandra and $\mathrm{XMM}$, above $\sim 5 \mathrm{keV}$; a more detailed discussion can be found in Worsley et al. (these proceedings). This is important, since the background spectrum, when plotted in the $E \times F(E)$ form, peaks somewhere near $30 \mathrm{keV}$. With this, it will be important to conduct sensitive observations in the hard X-ray band, and the currently planned hard X-ray imaging satellite NuSTAR has this as one of its main goals.

Chandra's accomplishments regarding various astrophysical sources, especially neutron stars, supernova remnants, and active galaxies, were discussed extensively at the Torun meeting and a number of results are discussed in various papers contained in these proceedings. In particular, well-known is the discovery of large-scale (kpc) X-ray emitting jets, especially in the highly luminous, distant quasars (see Fig. 1); this is discussed more extensively by Stawarz (these proceedings). There, the best scenario for production of X-ray radiation is Compton upscattering of the Cosmic Microwave Background radiation by energetic, relativistic electrons in the jet, but regardless of the radiation mechanism, the radiating particles must be accelerated locally. The data for extended AGN jets are likely to hold many clues as to the processes responsible for formation of 
these jets, their composition, as well as the transport of energy from the central source - presumably an accreting supermassive black hole - to the distances comparable (or larger) to the size of the host galaxy; this subject is discussed in Sikora et al. (these proceedings). Below I focus on one area not covered by other contributions, namely clusters of galaxies.

\section{Clusters of galaxies observed with Chandra and XMM}

The superb quality of the Chandra mirrors produced exquisite images of extended celestial X-ray sources, and this is certainly true for clusters of galaxies. The images revealed rich structure, associated with the process of formation and evolution of clusters - such as merging activity, or interaction of the central AGN with the intra-cluster medium. The availability of high-quality strong and weak lensing data allows detailed comparison of the gravitating mass against the properties of the X-ray emitting gas. In general, the total (dark + baryonic matter) masses inferred from weak lensing agree with those inferred from X-ray observations, but discrepancies betwen X-ray and strong lensing masses are commonly inferred in non-relaxed systems, which are presumably still forming, and are not in dynamical equilibrium. Particularly compelling results were inferred from the Chandra observations of the "bullet cluster" (1E0657-56; Fig. 2) by Markevitch et al. (2004) and Clowe et al. (2004). Those authors report that the cluster is undergoing a high-velocity $\sim 4500 \mathrm{~km} \mathrm{~s}^{-1}$ merger, evident from the spatial distribution of the hot, X-ray emitting gas, but this gas lags behind the subcluster galaxies. Furthermore, the dark matter clump, revealed by the weak-lensing map, is coincident with the collisionless galaxies, but lies ahead of the collisional gas. This and other similar observations - allow good (and interesting) limits on the cross-section of the self-interaction of dark matter. Observations such as this - and many other, similar ones - are bound to reveal the details of the process of cluster formation, and in particular, of the heating of the intra-cluster gas.

Another important aspect of the cluster evolution is the interaction of the central AGN, present in many clusters of galaxies, with the intergalactic gas. Particularly impressive are the Chandra images of the Perseus cluster, revealing a cavity as well as ripples, presumably due to the interaction of the outflow (jet?) from the active galaxy NGC 1275 with the ICM of the cluster (Fabian et al. 2003; see Fig. 3). Such interaction might be responsible for the absence of cool cores in the cluster X-ray data; those in turn were expected on theoretical grounds, since the characteristic cooling time of the cluster gas should be relatively rapid, certainly much shorter than Hubble time. Instead, the high spectral resolution XMM-Newton data revealed that the clusters are generally devoid of cold cores, but instead, those cores have temperatures that are typically $\sim 1 / 3$ of the cluster average (Peterson et al. 2003).

Chandra continues to conduct observations of clusters, and the goal is two-fold. On one hand, the spatially-resolved spectroscopy allows a good insight into the formation of clusters and their evolution. Besides answering the obvious question: "what are the processes that heat the intra-cluster gas?" - such data should reveal some clues as to the origin of non-thermal population of energetic (relativistic) particles, inferred 


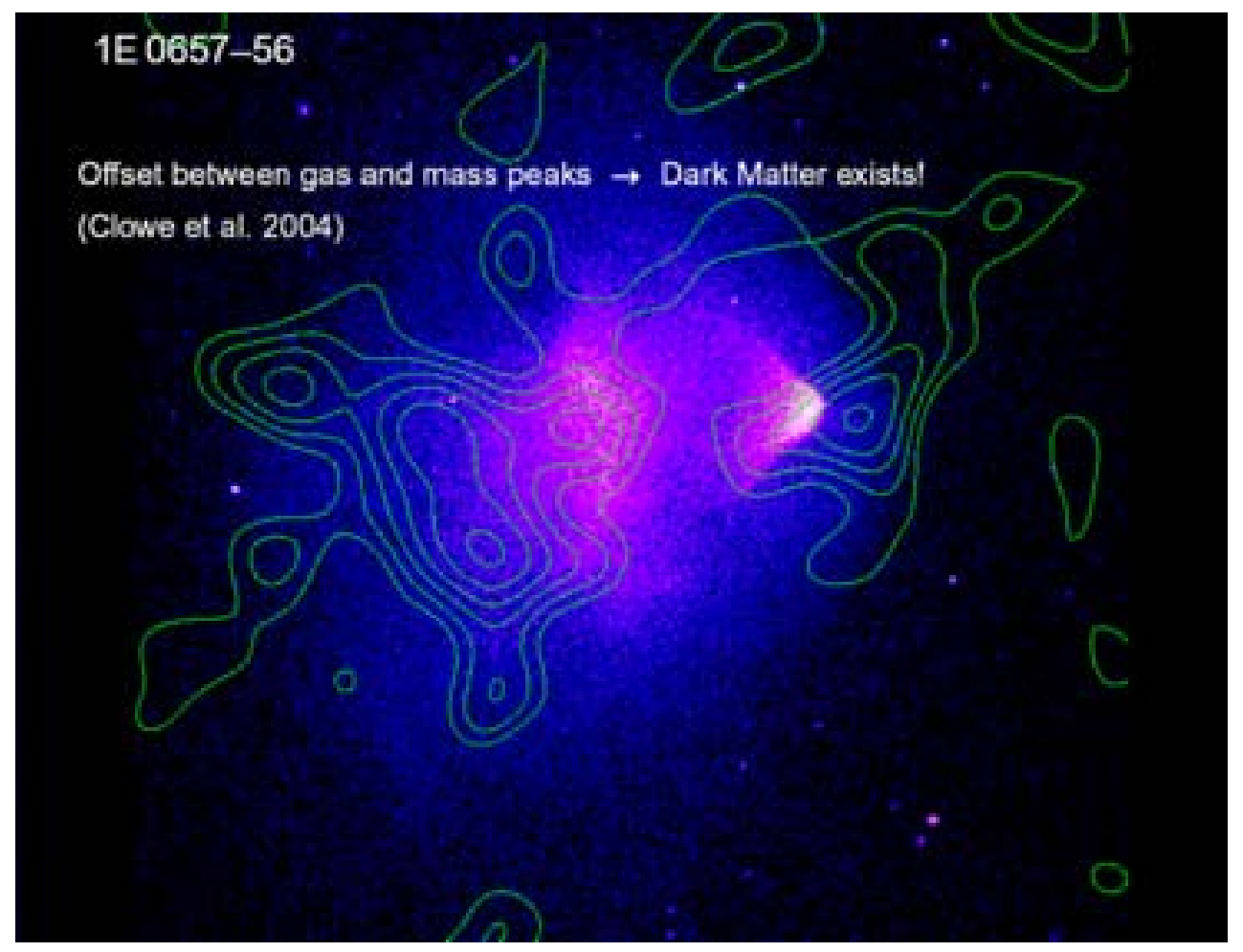

FIGURE 2. Chandra X-ray image of the "bullet cluster" (1E0657-56) overlaid on the inferred distribution of the matter - presumably associated with the cluster - that is likely responsible for lensing of light from background galaxies (figure courtesy of Maxim Markevitch, priv. comm., with permission). Data are from Markevitch et al. 2004 and Clowe et al. 2004. The offset between the X-ray - emitting matter and lensing matter distributions provides important evidence for existence of dark matter.

from radio observations. Radio emission is non-thermal, presumably due to synchrotron emission by relativistic particles in the intra-cluster magnetic field. Such particles might be accelerated in shocks, resulting from galaxy collisions during the formation of the cluster - and this is probably what the data for the "bullet cluster" (Fig. 2) reveal. If such energetic, non-thermal particles are indeed present, they should Compton-upscatter the Cosmic Microwave Background to hard X-ray energies. This should be detectable as a departure form thermal emission, and is best studied in the hard X-ray band, with instruments such as NuSTAR, described in subsequent sections.

Armed with good understanding of cluster evolution, we can use them as cosmological tools, since clusters should provide a "fair sample" of the matter content of the Universe. This very well complements the Cosmic Microwave Background and supernova data, providing somewhat orthogonal constraints towards the determination of cosmological parameters. There are several fruitful avenues to use the cluster data in cosmology. One includes measurements of absolute distances to clusters, providing the value of Hubble constant. This has been successfully attempted by a variety of workers, using Sunyaev-Zeldovich effect (see, e.g., Reese et al. 2004) or X-ray - inferred temperatures as well as masses of both dark an luminous matter (see, e.g., Allen et al. 2004). The other relies on determining the evolution of cluster density as a function of cosmological time, 


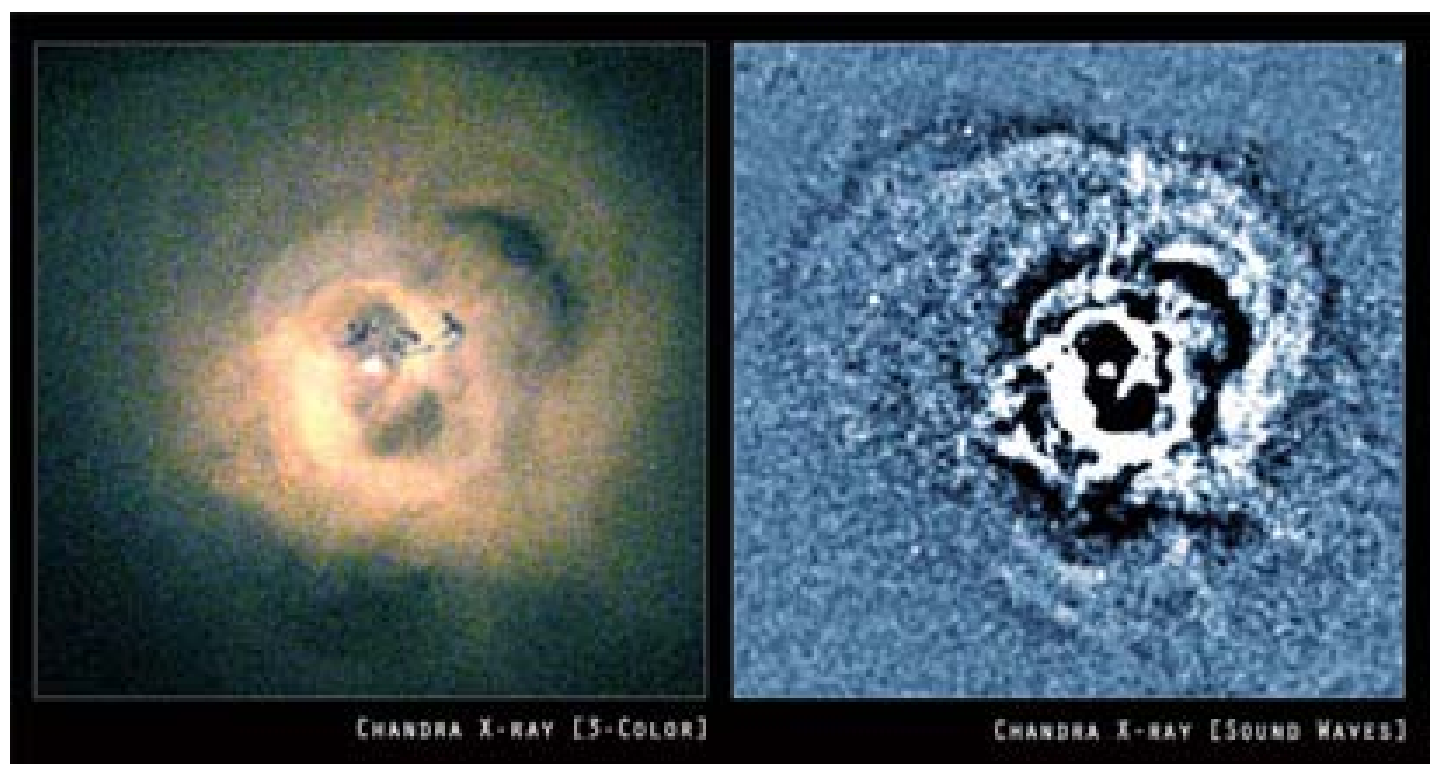

FIGURE 3. Deep Chandra X-ray image of the Perseus cluster (figure source: Chandra Science Center; figure credit: NASA; data published in Fabian et al. 2003). The left panel shows the smoothed image, while the right panel shows the image in high contrast. The structure seen in both images is real, presumably due to the interaction of the central AGN (NGC 1275) with the cluster gas.

and since this is related to the growth of structure in general, it depends sensitively on cosmological parameters. In both cases we need to measure the cluster masses, which is relatively straightforward if a cluster is dynamically relaxed but more challenging for the more complex, merging systems: this requires a good knowledge of the processes that lead to formation of the cluster.

\section{FUTURE HIGH-ENERGY ASTROPHYSICS MISSIONS}

While the current data are providing us with spectacular high-energy observations of celestial sources, many questions remain. Perhaps the most pressing is the dynamical state of the X-ray emitting gas in a wide range of celestial sources. This is best studied with high resolution X-ray spectroscopy, where the Doppler shifts of spectral features provide information about the kinematics of the gas. Another somewhat under-explored area regards the details of the hard X-ray and soft gamma-ray emission, bound to reveal the nature of the fundamental processes converting gravitational energy of material being accreted onto a compact object into radiation. This must be associated with heating and acceleration of particles to relativistic energies, and is best probed at energies beyond where the thermal processes dominate, which for many sources is around 10 $\mathrm{keV}$. Finally, the very successful EGRET instrument, operational in the 1990s on-board of the Compton Gamma-ray Observatory, revealed many different classes of energetic $(\sim \mathrm{GeV})$ gamma-ray emitters, but better understanding of those sources requires data with more sensitive instruments. The future appears promising: Suzaku should usher sensitive, high-resolution X-ray spectroscopy, with energy resolution at least 10 times 
better than the current "workhorses" of X-ray astronomy, CCDs; NuSTAR will, for the first time, provide us with X-ray optics for imaging data for celestial sources in the 10 $80 \mathrm{keV}$ band, improving the ultimate sensitivity by a factor of at least several hundred; and GLAST will have significantly improved sensitivity and broader bandpass than its predecessor EGRET. The capabilities of those three new missions are briefly discussed below.

\section{Suzaku (Astro-E2)}

This satellite is the next in a series of Japanese X-ray astronomy missions, following the successful Hakucho, Tenma, Ginga, and Asca, and will be launched about 2 weeks after the conclusion of the Torun conference. Developed in collaboration with US institutions, it features three types of instruments. The premier instrument, the Xray Spectrometer (XRS), is a non-dispersive, cryogenically cooled detector, operating at $\sim 60 \mathrm{mK}$, with the expected cryogen lifetime of $\sim 2.5$ years. The detector, developed by the Goddard Space Flight Center consists of 32 independent sensors (pixels), where the interaction of an X-ray with the sensor results in a temporary rise of the sensor temperature roughly proportional to the energy of the incoming X-ray. The XRS is located in the focal plane of an X-ray mirror with a modest (1 arc min) point-spread function. The mirror / detector combination provides sensitivity over the $0.3-10 \mathrm{keV}$ bandpass with effective area $>100 \mathrm{~cm}^{2}$ over the $0.8-8 \mathrm{keV}$ band, providing an energy resolution better than $10 \mathrm{eV}$. The primary objective of this instrument is to study extended (rather than point-like) sources such as supernova remnants and clusters of galaxies, which cannot be reliably studied with diffraction gratings such as those onboard XMM-Newton or Chandra. ${ }^{1}$

Besides the XRS, Suzaku features four more X-ray mirrors, each with the PSF of $\sim 1$ arc min, similar to the mirror for the XRS. Each mirror focuses X-rays onto an Xray Imaging Spectrometer (XIS) featuring a CCD chip (similar in performance to those flown on the Chandra satellite), covering $18 \times 18$ arc min, and featuring the effective area of $\sim 400 \mathrm{~cm}^{2}$ at $1.5 \mathrm{keV}$. The purpose of the XISs is to provide large effective area for detailed studies of temporally-resolved spectra, and will be particularly valuable after the depletion of the XRS cryogen. This capability, coupled with the relatively large solid angle subtended by the CCDs, will be useful for mapping of substantial regions of the sky, or for monitoring of flux and spectral variability of X-ray sources.

The third kind of instrument on-board Suzaku is the Hard X-ray Detector, a nonimaging, collimated device with effective area of nearly $200 \mathrm{~cm}^{2}$, sensitive over the $\sim 8-700 \mathrm{keV}$ range. It actually consists of two separate sets of sensors: the energy range $\sim 8-50 \mathrm{keV}$ is covered by PIN silicon diodes (with the field of view of $1 / 2 \times 1 / 2$ degree), while over $50-700 \mathrm{keV}$, the sensors are GSO scintillators (with the field of view of $\sim 4 \times 4$ degrees), physically located behind the PIN diode, which becomes transparent to X-rays with $E>50 \mathrm{keV}$. The detector features a well-type design, meaning that the

\footnotetext{
${ }^{1}$ Unfortunately, as these proceedings were undergoing the editorial process, the cryogenic system, responsible for cooling the XRS, failed and resulted in the loss of the cryogen.
} 


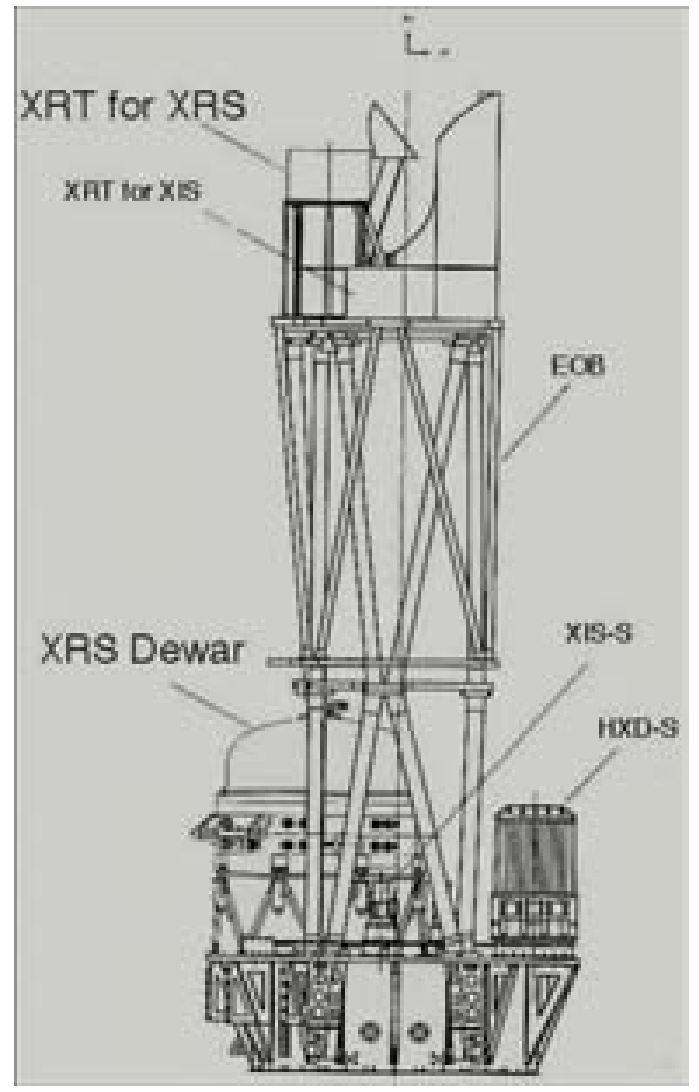

FIGURE 4. Schematic illustrations of the Suzaku satellite, indicating its instruments: the X-ray Spectrometer (XRS), the X-ray Imaging Spectrometer (XIS), and the Hard X-ray Detector (HXD). XRT refers to the X-ray Telescope, and EOB to the Extendable Optical Bench. Figure credit: JAXA/ISAS

collimator (as well as the back and the sides of the instrument) are constructed of anticoincidence shield, dramatically reducing the particle-induced background.

More detailed description of Suzaku instruments as well as full references can be found at http://heasarc.gsfc.nasa.gov/docs/astroe/

\section{GLAST}

Gamma-Ray Large Area Space Telescope (GLAST) is under construction for a launch in 2007. Its goal is to follow the successful energetic gamma-ray detector EGRET flown on-board of the Compton Gamma-Ray Observatory towards a study of celestial sources of gamma-ray radiation in the $30 \mathrm{MeV}-300 \mathrm{GeV}$ range. Such a broad range, especially at the high energy end, should allow some overlap of sensitivity with the groundbased Cherenkov telescope arrays, a feature highly desirable for cross-calibration. The main instrument, the Large Area Telescope (LAT), is essentially a very sophisticated particle tracker, and is currently undergoing integration and test at the Stanford Linear Accelerator Center. It determines the direction and energy of an incident gamma-ray by 


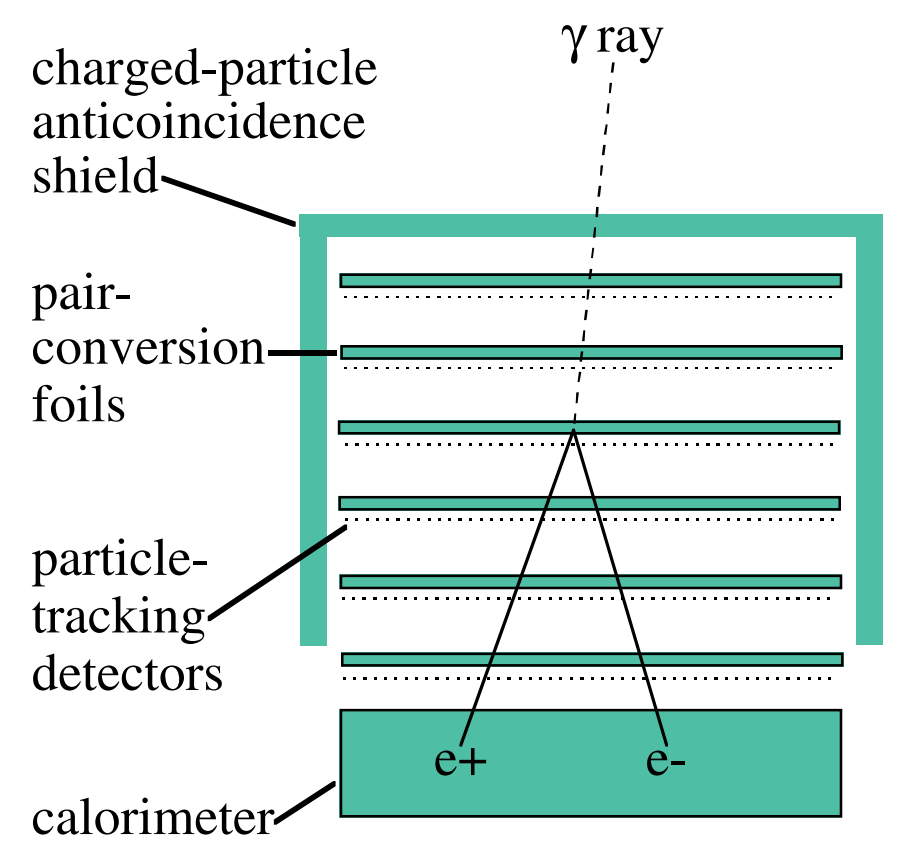

FIGURE 5. Schematic illustration of the principle of operation of the Large Area Telescope, the main instrument onboard of the GLAST satellite. The calorimeter is capable of determining the total energy of particles resulting from the interaction of the gamma-ray with the converter, and should allow for energy resolution of the instrument of $\sim 10 \%$. Figure credit: NASA and SLAC

tracking the products of the interaction of the gamma-ray with converter material; the particle tracking uses silicon strip detectors. The tremendous increase in sensitivity (as compared to EGRET) is mainly due to three separate improvements. One is the larger effective area of the instrument, which, after accounting for instrumental inefficiencies should be at least $8000 \mathrm{~cm}^{2}$ at $1 \mathrm{GeV}$. Another is the fact that the detector has wide angle of acceptance, simultaneously "seeing" about 2 steradians of the sky. Finally, the third improvement is the significantly better ability to localize the direction of the incoming gamma-rays, which in turn allows for fewer background events or truly diffuse gammarays in the region corresponding to the PSF. The current design goals call for the on-axis Point-Spread Function of $<3.5$ degrees at $100 \mathrm{MeV}$ and $<0.15$ degrees at $10 \mathrm{GeV}$ (localization of celestial sources should be considerably better than these values, via centroiding).

The LAT detector will be always pointing away from the Earth, thus maximizing the exposure to the celestial gamma-rays. With the large solid angle of acceptance of 2 steradian, the GLAST LAT is particularly well-suited to monitoring of gamma-ray flux from celestial sources, since every patch of the sky will be in the field of view of the detector for at least a fraction of each day. This will be particularly useful for studies of variable gamma-ray sources such as the jet-dominated active galactic nuclei (blazars) that proved to be the most numerous point-like sources in the EGRET data. However, since any better understanding of those sources will require observations at many different spectral bands, the real challenge will be to obtain adequate radio, IR, 


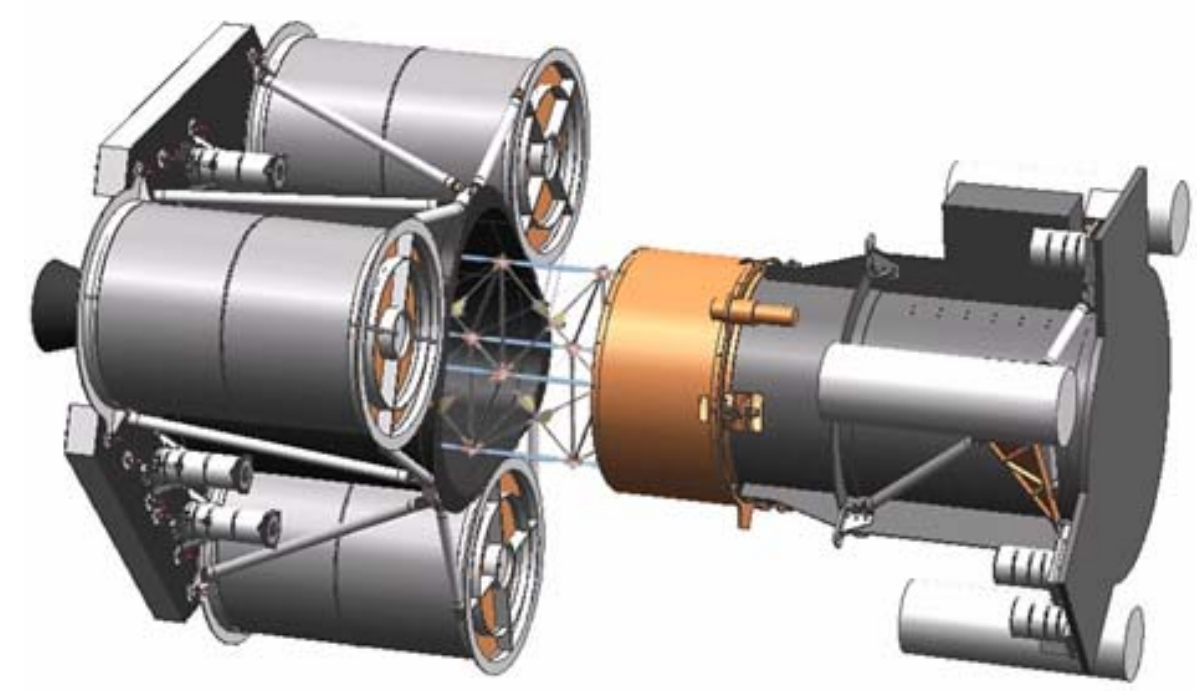

FIGURE 6. Schematic illustrations of the NuSTAR satellite, designed to image the celestial X-ray sources in the hard X-ray band. The payload will extend in orbit, allowing for 10 meter separation between the X-ray mirrors and detectors. Figure credit: F. Harrison, NuSTAR PI, and NASA/JPL

optical, and X-ray monitoring for a good number of sources, since all those bands generally allow studies of celestial sources "one at a time." Still, at least in the X-ray band, the Suzaku above, and NuSTAR below, are very well-suited to this task.

\section{NuSTAR}

While the improvement in sensitivity of astrophysical instruments operating at energies below $10 \mathrm{keV}$ has been remarkable, mainly via the Chandra and XMM-Newton observatories, above $10 \mathrm{keV}$, recent progress was only modest. This is mainly due to experimental limitations: the in-orbit background (both cosmic X-ray, and particle-induced) is considerable, and thus increasing the area of a detector for simple, non-imaging, collimated instruments, is only of limited use. To achieve a large sensitivity gain, it is necessary to image the sky, preferably via the use of X-ray focusing optics. This approach is of course the reason for the dramatic improvements below $10 \mathrm{keV}$ : it allows a reduction of the size of the detector (thus reducing background) but still, allows the collecting area - that of the telescope - to be only limited by the cost.

Above $10 \mathrm{keV}$, the recent major breakthrough is the application of multi-coated layers to grazing incidence X-ray optics, and this technique allows for focusing of hard X-rays. Success of this approach has been demonstrated by the recent flight of the Caltech - Livermore - Columbia - DSRI balloon payload HEFT (Harrison et al. 2000). This payload included a mirror capable of focusing hard X-rays onto a CdZnTe pixellated detector, with energy resolution of $\sim 1 \mathrm{keV}$. A similar approach is planned for the satellite-based instrument NuSTAR, also developed by the HEFT team but also including Stanford, UCSC, and JPL: it will feature three multi-coated grazing incidence telescopes capable of focusing X-rays with energies 10 up to $80 \mathrm{keV}$ onto 
detectors similar to those flown on HEFT. Since the grazing incidence angles required for reflection at the hard X-ray energies are shallow, a long focal length is required for an appreciable collecting area of the mirrors. To this end, NuSTAR will feature an extendible optical bench, allowing for a $10 \mathrm{~m}$ focal length, even though the total payload in the launch configuration will be no more than $\sim 1 \times 1 \times 1$ meter. The anticipated total effective area of all three telescope/detector systems is at least $300 \mathrm{~cm}^{2}$ at $30 \mathrm{keV}$, with the point-spread function of $40^{\prime \prime}$. This will result in a dramatic reduction of background, and will improve the sensitivity by a factor of many hundreds as compared with the previously flown, non-imaging hard X-ray instruments.

NuSTAR will fly in 2009 as a part of NASA's Small Explorer program. It has three main science goals: first, to take the census of black holes at all sizes. This includes those powering active galaxies that contribute to the Cosmic X-ray background - and hopefully will result in resolving the questions brought by the current $E<10 \mathrm{keV}$ data (see, e.g., Worsley et al., these proceedings). Another goal is to study the process of elemental enrichment of the Universe, by measuring spectra of young supernova remnants in a search for ${ }^{44} \mathrm{Ti}$ emission lines: the content and kinematics of this element in the ejecta has important implication on the details of supernova explosion. Finally, NuSTAR will work closely with GLAST, providing the unique capability of monitoring the hard X-ray flux from the gamma-ray emitting variable celestial sources such as the jet-dominated active galaxies. This is only a short list of scientific goals: others include search for, and mapping the continuum hard X-ray emission in clusters of galaxies (revealing the details of non-thermal particle population), supernova remnants (in search for the origin of most energetic particles accelerated in the SNR and related to the Galactic cosmic rays).

In summary, the "golden era" of high energy astrophysics, ushered by great instruments and resulting in spectacular discoveries, is likely to continue for many years to come.

\section{REFERENCES}

1. Allen, S., et al. MNRAS, 353, 457 (2004)

2. Clowe, D., Gonzalez, A., and Markevitch, M. ApJ, 604, 596 (2004)

3. Fabian, A., et al. MNRAS, 344, 43 (2003)

4. Harrison, F., et al. Proc. SPIE 4012, 693 (2000)

5. Markevitch, M., et al. ApJ,606, 819 (2004)

6. Peterson, J., et al. ApJ, 590, 207 (2003)

7. Reese, E., et al. ApJ, 581, 53 (2002)

8. Schwartz, D. A., et al. ApJL, 540, 69 (2000)

9. Wilson, A., Young, A., and Shopbell, P. ApJL, 544, 27 (2000) 